Tandem pulsed acousto-optics: obtaining the tagged light fraction from modulated non-ideal speckle patterns

This content has been downloaded from IOPscience. Please scroll down to see the full text. 2016 Phys. Med. Biol. 61504

(http://iopscience.iop.org/0031-9155/61/2/504)

View the table of contents for this issue, or go to the journal homepage for more

Download details:

IP Address: 130.89.45.232

This content was downloaded on 22/09/2016 at $12: 12$

Please note that terms and conditions apply. 


\title{
Tandem pulsed acousto-optics: obtaining the tagged light fraction from modulated non-ideal speckle patterns
}

\section{S G Resink and W Steenbergen}

Biomedical Photonic Imaging group, MIRA Institute for Biomedical Technology and Technical Medicine, University of Twente, PO Box 217, 7500 AE Enschede, The Netherlands

E-mail: w.steenbergen@utwente.nl

Received 25 May 2015, revised 7 November 2015

Accepted for publication 20 November 2015

Published 18 December 2015

\begin{abstract}
Recently we presented novel methods for acousto-optic (AO) imaging of biological tissues, taking (1) the mean square difference of speckle patterns (subtraction method) or (2) the contrast of the summation of speckle patterns (summation method) acquired from nanosecond pulses of coherent light, fired at different ultrasound phases. In this study we relate the two methods both analytically and experimentally. We experimentally show that these two methods are nearly identical provided that the maximum achievable speckle contrast is determined correctly. We show with simulations that after correction the outcome is independent of experimental detection parameters. This makes the AO methods in this study reliable, allowing quantifying speckle observations in terms of the ultrasonically tagged fractions of light. The use of tandem nanosecond pulses in one burst of ultrasound overcomes the challenge of tissue dynamics.
\end{abstract}

Keywords: acousto-optics, speckle, speckle dynamic, speckle contrast

(Some figures may appear in colour only in the online journal)

\section{Introduction}

Photoacoustics (PA) and acousto-optic tomography (AOT) (Resink et al 2012) combine the use of sound and light in turbid media. The combination of PA and AOT measurements makes 
it possible to perform fluence compensated photoacoustic imaging as shown by Daoudi et al (2012) and Hussain et al (2014). In this approach, the so called ultrasonically modulated or 'tagged' fraction of light plays an important role. Ideally, the measurements are done with the same laser system, This reduces the costs, increases acquisition speed and gives more reliable results. However, these techniques have different laser requirements. PA needs short (typically $\sim 5 \mathrm{~ns}$ ) high energy ( $>\mathrm{mJ}$ ) pulses while acousto-optics (AO) needs a long coherence length $(>1 \mathrm{~m})$ and a temporal behavior that allows recording of the dynamic behavior of speckles under the influence of ultrasound. Recently we have shown the possibility to perform AO imaging with a laser system also suitable for PA (Resink et al 2014b), in a manner that overcomes the problem of tissue dynamics (Resink et al 2014a).

$\mathrm{AO}$ measurements are usually performed using a quasi CW laser so that a camera, or other optical detector, can integrate over multiple US cycles. The cameras are used for their high number of optical detector elements and the increase in SNR that results from acquiring information from a great number of speckles (Leveque et al 1999).

One AO method that relies on a camera is the speckle contrast method (Li et al 2002). However, we use a nanosecond laser pulse and therefore, no information on speckle dynamics is obtained within the laser pulse duration. The camera captures an unblurred speckle pattern that ideally has a unity contrast. However, it is possible to perform a speckle contrast measurement by integrating over multiple optical pulses applied in a range of ultrasound phases. In this study, we investigate two methods both experimentally and analytically: one is referred to as subtraction method while the second one is referred to as the summation method where two speckle patterns generated by nanosecond laser pulses are subtracted or added respectively. For these approaches, Resink et al (2015) recently presented a theoretical framework in the case of ideal speckle patterns with unity contrast. Unfortunately, in experiments the contrast is less than unity because of noise, background radiation and imperfect source coherence. Therefore we need an expression that translates the AO signals obtained from non-ideal speckle patterns into signals as they would have been measured if the speckle pattern had a contrast close to unity Here we present a theoretical framework to extract tagged fractions in case of non-ideal speckle patterns, hence with a background signal and noise, and compare this with the well known speckle contrast method (Li et al 2002, Zemp et al 2006). Further we show that the data reproduces correct $\mathrm{AO}$ values that are independent from the speckle contrast. This finding makes it possible to perform pulsed $\mathrm{AO}$ in a more quantitative way.

\section{Theory}

\subsection{Relationship between the summation and subtraction methods in $A O$}

Speckle pattern subtraction would require two separate speckle patterns to be recorded, either within one US cycle, or at least far within the speckle decorrelation time, e.g. using a high speed camera. However, by recording two speckle patterns within the same exposure of a slow camera it is possible to add instead of subtract the speckle patterns. This way the two laser pulses can be fired without inducing speed constraints on the used camera. Because of the US-induced changes in the speckle pattern, the sum of the two speckle patterns has a lower contrast than a singe speckle contrast. We call this procedure the summation method. The summation of the speckle patterns is similar to the speckle contrast method derived earlier (Li et al 2002, Resink et al 2015), except that the integration of light is now only carried out for two phases of the ultrasound instead of a continuum over $2 \pi$ phases. In both summation and contrast method, the difference in contrast can be related to the tagged fraction. As shown by Resink et al (2015) the temporal behavior of the intensity of the modulated speckles is described by 


$$
I(t)=I_{0}+I_{\omega}(t)+I_{2 \omega}(t)
$$

with $I_{0}$ the static component, $I_{\omega}$ and $I_{2 \omega}$ are dynamic and have a frequency of the US and twice that of the US respectively. The subtraction and summation methods each use different terms from equation (1). $I_{0}$ is static and thus would appear in a sum image twice. $I_{\omega}$ has an opposite sign at $\pi$ phase and therefore this term cancels out on addition and does not contribute to the resultant signal of the addition method. The harmonic term appears out of the multiplication of the electric fields associated with the two side bands that form the tagged light. The average value of $I_{2 \omega}$ is zero and doesn't contribute to the average intensity. However, the standard deviation of $I_{2 \omega}$ is non zero. Spatial averaging reduces the influence of $I_{2 \omega}$ to 0 and is therefore ignored in the rest of our analysis. However the noise from this term will be visible in the signal, so the summation method is similar to the contrast method but not the same.

We want to adapt both methods for more realistic cases where the maximum speckle contrast is less than unity. In these cases the speckle contrast reduction is normalized with the maximum speckle contrast. This method is also used in laser speckle contrast methods for perfusion imaging (LSCI) (Briers et al 2013) to make results more comparable between different experiments and setups. How this correction influences the estimation of the tagged fraction is given in the next section.

In both the summation and subtraction method, two speckle patterns are recorded; one at phase $0: I(\vec{x}, 0)$ the other at time $\pi / \omega: I(\vec{x}, \pi / \omega)$.

A speckle pattern $I(\vec{x}, t)$ can be written as its average value $\langle I(\vec{x}, t)\rangle$ plus a deviation $\Delta(\vec{x}, t)$ from that average. For simplicity we assume the speckle patterns are normalized and thus the average value is unity, leading to

$$
I(\vec{x}, t)=1+\Delta(\vec{x}, t)
$$

The two speckle patterns are very similar but with small differences that are induced by the ultrasound. They can be written as

$$
\begin{aligned}
& I(\vec{x}, 0)=1+\Delta(\vec{x}, 0) \\
& I(\vec{x}, \pi / \omega)=1+\Delta(\vec{x}, \pi / \omega)=1+f(\Delta(\vec{x}, 0), \varepsilon)
\end{aligned}
$$

where $f(\Delta(\vec{x}, t), \varepsilon)$ is an unknown function that gives $\Delta(\vec{x}, \pi / \omega)$ as function of $\Delta(\vec{x}, 0)$ and small differences of the optical pathlengths $\varepsilon$ induced by the ultrasound. The use of this function allows that some speckles become brighter and some darker and thus resembles the differences of a speckle pattern induced by the ultrasound. The properties of function $f(\Delta(\vec{x}, 0), \varepsilon)$ that we know are

$$
\begin{aligned}
& \langle f(\Delta(\vec{x}, 0), \varepsilon)\rangle=0 \\
& \left\langle f(\Delta(\vec{x}, 0), \varepsilon)^{2}\right\rangle=c_{\max }^{2}
\end{aligned}
$$

In which $<>$ denotes spatial averaging, $c_{\max }$ the maximum achievable speckle contrast.

Starting with the relation derived in (Resink et al 2015) between the tagged fraction $R_{\text {diff }}$ and the differences between the speckle patterns, we can write:

$$
\begin{aligned}
R_{\mathrm{diff}} & \approx \frac{1}{8}\left\langle(I(\vec{x}, 0)-I(\vec{x}, \pi / \omega))^{2}\right\rangle \\
& =\frac{1}{8}\left\langle(\Delta(\vec{x}, 0)-f(\Delta(\vec{x}, 0), \varepsilon))^{2}\right\rangle \\
& \frac{1}{4}\left(c_{\max }^{2}-\langle\Delta(\vec{x}, 0) f(\Delta(\vec{x}, 0), \varepsilon)\rangle\right)
\end{aligned}
$$

To make this number independent of the speckle contrast we normalize with $c_{\max }^{2}$. 


$$
\begin{aligned}
R_{\text {diff,Norm }} & =\frac{\left\langle(I(\vec{x}, 0)-I(\vec{x}, \pi / \omega))^{2}\right\rangle}{8 c_{\max }^{2}} \\
& =\frac{1}{4}-\frac{\langle\Delta(\vec{x}, 0) f(\Delta(\vec{x}, 0), \varepsilon)\rangle}{4 c_{\max }^{2}}
\end{aligned}
$$

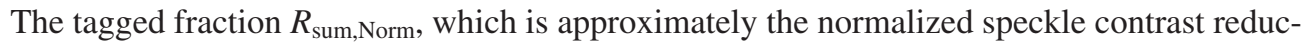
tion for small tagged fractions (Resink et al 2015), derived from the normalized speckle contrast from a two speckle pattern summation is given by (Resink et al 2014a):

$$
\begin{aligned}
R_{\text {sum, norm }} & \approx 1-\frac{\sqrt{\frac{\left\langle(I(\vec{x}, 0)+I(\vec{x}, \pi / \omega))^{2}\right\rangle}{\langle I(\vec{x}, 0)+I(\vec{x}, \pi / \omega)\rangle^{2}}-1}}{c_{\max }} \\
& =1-\frac{\sqrt{\frac{\left\langle(2+\Delta(\vec{x}, 0)+f(\Delta(\vec{x}, 0), \varepsilon))^{2}\right\rangle}{4}-1}}{c_{\max }} \\
& =1-\frac{\sqrt{\frac{\left.4+\left\langle\Delta(\vec{x}, 0)^{2}\right\rangle+\langle f(\Delta(\vec{x}, 0), \varepsilon))^{2}\right\rangle+2\langle\Delta(\vec{x}, 0) f(\Delta(\vec{x}, 0), \varepsilon)\rangle}{4}-1}}{c_{\max }} \\
& =1-\frac{\sqrt{\frac{1}{2} c_{\max }^{2}+\frac{1}{2}\langle\Delta(\vec{x}, 0) f(\Delta(\vec{x}, 0), \varepsilon)\rangle}}{c_{\max }} \\
& \approx 1-\frac{c_{\max }+\frac{\langle\Delta(\vec{x}, 0) f(\Delta(\vec{x}, 0), \varepsilon)\rangle}{4 c_{\max }}-\frac{c_{\max }}{4}}{c_{\max }} \\
& =\frac{1}{4}-\frac{\langle\Delta(\vec{x}, 0) f(\Delta(\vec{x}, 0), \varepsilon)\rangle}{4 c_{\max }^{2}}
\end{aligned}
$$

The identity of equations (6) and (7) shows that the sum and subtraction methods give the same tagged fraction. However both methods are based on different terms of equation (1) that clearly contain the same information. Both methods suggest a normalization by the speckle contrast in the situation where there is no tagging. The validity of this normalization is investigated in the next paragraphs. It will appear that through proper normalization, the results of the subtraction and sum methods can quantitatively equivalent.

\subsection{Correcting the estimations for non-ideal speckle patterns}

Non-ideal and ideal speckle patterns differ in various ways. First, when we add a DC offset to the speckle pattern the speckle contrast is reduced. Next, the spatial sampling of the speckle pattern also influences the observed speckle contrast. The speckle contrast approaches unity when taking the limit of the number of pixels per speckle toward infinity (Ramirez-San-Juan et al 2013). The finite size of a camera pixel also reduces the speckle contrast because the measured intensity by the pixel is spatially averaged (Thompson et al 2011). To overcome these problems in LSCI it is common to normalize the speckle contrast with the maximum achievable speckle contrast for the given setup. It was shown that this approach works in LSCI (Thompson et al 2011) and in this study we show that it also works in $\mathrm{AO}$, and that this procedure gives the correct estimation for the tagged fraction defined in Resink et al (2015). 
$I_{t, \text { ideal }}$ is a speckle pattern that has a contrast for small tagged fractions $R$ given by a model as: $c_{t, \text { ideal }} \approx 1-R$ (Resink et al 2015$)$. From here on we omit the use of spatial coordinate $\vec{x}$ in the equations for readability.

We write the observed nonideal speckle pattern $I_{\text {measured }}$ as the sum of an ideal tagged speckle pattern $I_{t, \text { ideal }}$ and a speckle contrast distorting function $\gamma$, giving

$$
I_{\text {measured }}=I_{t, \text { ideal }}+\gamma
$$

The resulting speckle contrast is thus for a normalized speckle pattern

$$
\begin{aligned}
c_{\text {measured }}^{2} & =\left\langle I_{\text {measured }}^{2}\right\rangle-1 \\
& =\left\langle\left(I_{t, \text { ideal }}+\gamma\right)^{2}\right\rangle-1
\end{aligned}
$$

This measured speckle pattern is corrected by normalizing it with the maximum contrast:

$$
\begin{aligned}
c_{\text {corrected }}^{2} & =\frac{c_{\text {measured }}^{2}}{c_{\text {max }}^{2}} \\
& =\frac{\left\langle\left(I_{t, \text { ideal }}+\gamma\right)^{2}\right\rangle-1}{\left\langle\left(I_{u, \text { ideal }}+\gamma\right)^{2}\right\rangle-1} \\
& =\frac{\left\langle I_{t, \text { ideal }}^{2}\right\rangle+\left\langle\gamma^{2}\right\rangle+2\left\langle I_{t, \text { ideal }} \gamma\right\rangle-1}{\left\langle I_{u, \text { ideal }}^{2}\right\rangle+\left\langle\gamma^{2}\right\rangle+2\left\langle I_{u, \text { ideal }} \gamma\right\rangle-1}
\end{aligned}
$$

where $I_{u \text {,ideal }}=I_{t, \text { ideal }}$ if the tagged fraction is zero. When the average value of both $I_{t, \text { ideal }}$ and $I_{u \text {,ideal }}$ is the same, thus $\left\langle I_{\mathrm{t}, \text { ideal }}\right\rangle=\left\langle I_{u \text {,ideal }}\right\rangle$ then from the definition of the speckle contrast the relation between $c_{t, \text { ideal }}$ and the two speckle patterns is given as:

$$
\begin{aligned}
\left\langle I_{t, \text { ideal }}^{2}\right\rangle & =\left\langle I_{u, \text { ideal }}\right\rangle^{2}\left(c_{t, \text { ideal }}^{2}+1\right) \\
\left\langle I_{u, \text { ideal }}^{2}\right\rangle & =2\left\langle I_{u, \text { ideal }}\right\rangle^{2}
\end{aligned}
$$

By substituting this in equation (10) we obtain

$$
c_{\text {corrected }}^{2}=\frac{\left\langle I_{u, \text { ideal }}\right\rangle^{2}\left(c_{t, \text { ideal }}^{2}+1\right)+\left\langle\gamma^{2}\right\rangle+2\left\langle I_{t, \text { ideal }} \gamma\right\rangle-1}{2\left\langle I_{u, \text { ideal }}\right\rangle^{2}+\left\langle\gamma^{2}\right\rangle+2\left\langle I_{u, \text { ideal }} \gamma\right\rangle-1}
$$

We are interested in speckle contrast differences $\Delta c$ between modulated and non-modulated speckle patterns, which for small values can be related to $c$ as

$$
\begin{aligned}
c^{2} & =(1-\Delta c)^{2} \\
& =1-2 \Delta c+\Delta c^{2} \\
& \approx 1-2 \Delta c
\end{aligned}
$$

Substituting equation (13) in equation (12) gives

$$
1-2 \Delta c_{\text {corrected }}=\frac{\left\langle I_{u, \text { ideal }}\right\rangle^{2}\left(2-2 \Delta c_{t, \text { ideal }}\right)+\left\langle\gamma^{2}\right\rangle+2\left\langle I_{t, \text { ideal }} \gamma\right\rangle-1}{2\left\langle I_{u, \text { ideal }}\right\rangle^{2}+\left\langle\gamma^{2}\right\rangle+2\left\langle I_{u, \text { ideal }} \gamma\right\rangle-1}
$$

Because all speckle patterns in this study are normalized we can write the following relations 


$$
\begin{aligned}
& \left\langle I_{t, \text { ideal }}+\gamma\right\rangle^{2}=1=\left\langle I_{t, \text { ideal }}\right\rangle^{2}+\langle\gamma\rangle^{2}+2\left\langle I_{t, \text { ideal }} \gamma\right\rangle \\
& \left\langle I_{u, \text { ideal }}+\gamma\right\rangle^{2}=1=\left\langle I_{u, \text { ideal }}\right\rangle^{2}+\langle\gamma\rangle^{2}+2\left\langle I_{u, \text { ideal }} \gamma\right\rangle
\end{aligned}
$$

Because $\left\langle I_{\text {t,ideal }}\right\rangle^{2}=\left\langle I_{u, \text { ideal }}\right\rangle^{2}$ we obtain

$$
2\left\langle I_{t, \text { ideal }} \gamma\right\rangle=2\left\langle I_{u, \text { ideal }} \gamma\right\rangle=1-\left\langle I_{u, \text { ideal }}\right\rangle^{2}-\langle\gamma\rangle^{2}
$$

Therefore, we can simplify equation (14) by substituting this relation to obtain

$$
\Delta c_{\text {corrected }}\left(1+\frac{\left\langle\gamma^{2}\right\rangle-\langle\gamma\rangle^{2}}{\left\langle I_{u, \text { ideal }}\right\rangle^{2}}\right)=\Delta c_{t, \text { ideal }}
$$

Thus when the standard deviation of $\gamma$ is small compared with the intensity of the ideal speckle pattern, the $\Delta c$ after correction and the one predicted by the model are the same. This is the case for small errors induced by the finite size of pixels and for DC offsets. The measured contrast becomes higher when $\gamma$ represents noise on the camera. When the SNR of the measured speckle pattern is high the standard deviation of $\gamma$ is small compared to the average speckle intensity.

When we substitute a speckle pattern for $\gamma$ that represents a second polarization, and $\gamma$ thus depends on the tagged fraction like $I_{t, \text { ideal }}$ we have to derive this equation again in a similar way but we can now make the additional substitution $\left\langle\gamma^{2}\right\rangle=\langle\gamma\rangle^{2}\left(c_{t, \text { ideal }}^{2}+1\right)$ in equation (10). This substitution results in

$$
\Delta c_{\text {corrected }} \approx \Delta c_{t, \text { ideal }}
$$

Both equations (17) and (18) show that $\Delta c_{t, \text { ideal }}$ and $\Delta c_{\text {corrected }}$ are very similar and thus that correction by normalizing over the maximum obtainable speckle contrast not only makes the measurement independent of the speckle contrast but also gives numerical values that satisfy the behavior of an ideal modulated speckle pattern as defined in (Resink et al 2015). Thus the first line of equation (6) and the first line of equation (7) give us the tagged fraction independent of the maximum speckle contrast for the subtraction and summation method. The maximum speckle contrast of the setup can be determined performing a measurement without ultrasound or on a static turbid sample.

\section{Materials and methods}

The similarity of equations (17) and (18) is tested experimentally. Therefore we use the setup and experimental results of (Resink et al 2014b), see figure 1. Briefly, the experimental setup consists of an injection seeded frequency doubled Nd:YAG laser with a pulse repetition rate of $10 \mathrm{~Hz}$, wavelength of $532 \mathrm{~nm}$ and a Fourier limited pulse of $5 \mathrm{~ns}$ and a coherence length of approximately $1.5 \mathrm{~m}$. We inject 5 cycles of $5 \mathrm{MHz}$ ultrasound. Speckle patterns were recorded with a camera that was placed behind the sample. Because of the repetition rate of the laser we sample 2 speckle patterns with $100 \mathrm{~ms}$ time separation. By timing the injection of ultrasound with the laser we make sure that the sound burst was at the optical plane when the laser fires and has either a 0 or $\pi$ phase shift. An agar-intralipid phantom with $\mu_{\mathrm{s}}^{\prime}=0.6 \mathrm{~mm}^{-1}$ was used. This phantom is a $20 \mathrm{~mm}$ diameter cylinder making the $3 \mathrm{~mm}$ diameter optodes $20 \mathrm{~mm}$ apart.

The relation between the summation and the subtraction method is then verified from the experimentally obtained speckle patterns. The data used for the verification was presented in 


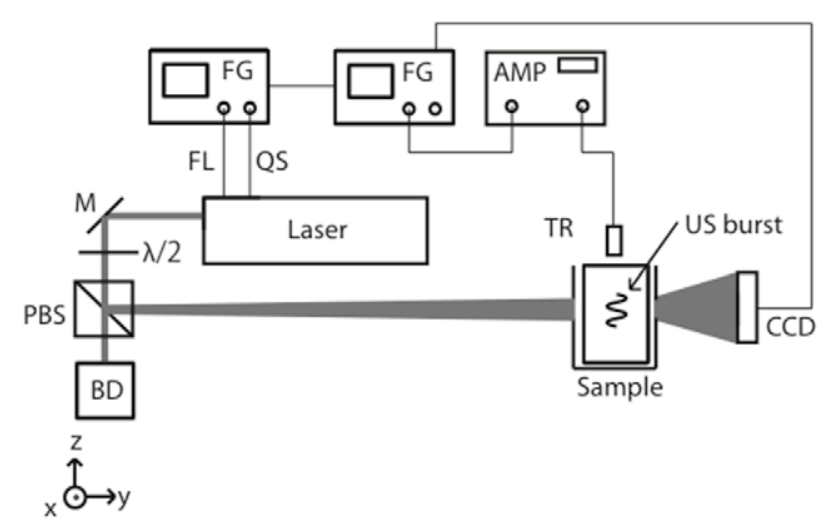

Figure 1. Experimental set-up. FG: function generators, AMP: amplifier, TR: ultrasound transducer and BD: beam dump.

(Resink et al 2014b) without the correction for the subtraction method. We performed a spatial scan in the plane perpendicular to the US propagation vector and between the optodes with a step size of $0.5 \mathrm{~mm}$.

We also performed numerical simulations to show the validity of equations (17) and (18). For this purpose we constructed several modulated speckle patterns. The contrast of a speckle pattern that is influenced by a DC offset, finite pixel size or extra polarizations was investigated. We used a $300 \times 300$ pixel reference speckle pattern where the speckles are 3 pixels in size. These patterns are the result of 2500 interfering phasors that originate from random points within an aperture having a random and modulated phase. This approach is similar to the one we used in (Resink et al 2015).

We numerically simulated two combined cases: one extreme and one with more realistic values. All these simulations were repeated 15 times so that the standard deviation can be plotted as error bar. We set a tagged fraction $R=0.01$ where all the phasors had the same phase modulation amplitude. The values obtained were corrected by normalizing by $c_{\max }$ for the summation and speckle contrast method (first step in equation (7)) and $c_{\max }^{2}$ for the subtraction method (equation (6)). Several distortions can act on a speckle pattern to lower the speckle contrast, the case without any distortions was our reference. Therefore we tested our method for the summation of a DC value with a magnitude of 1,2, or 3 times the average intensity of the speckle pattern ('DC1', 'DC2' and 'DC3'). Further, a second polarization lowered the speckle contrast ('2 Pol'). The speckle size in terms of pixels per speckle (P/S) was 0.7 Pixels/ Speckle ('0.7 P/S'), and 13 Pixels/Speckle ('13 P/S') compared with the 3 pixels per speckle of the reference to explore the effect of the amount of statistically independent data available while using the same number of pixels. The Shannon-Nyquist criterion is at 2 pixels per speckle. Further the finite size of pixels in a real camera lowers the contrast because of spatial averaging of small parts of the speckle pattern, this was simulated by convolving the speckle pattern with a square matrix of size $N(N=3,5,7)$ where all elements where equal to 1 . ('Blur 3', 'Blur 5', and 'Blur 7'). The simulation called 'All E' gave an extreme case of combined distortion, so 3 times the average value for the DC offset and 2 polarizations and 3 pixels per speckle and a blur matrix size of $7 \times 7$. And a more realistic case of combined distortions ('All R') was considered where the DC value was equal to the average speckle value (DC1) and 2 polarizations and a blur matrix size of 3 . 

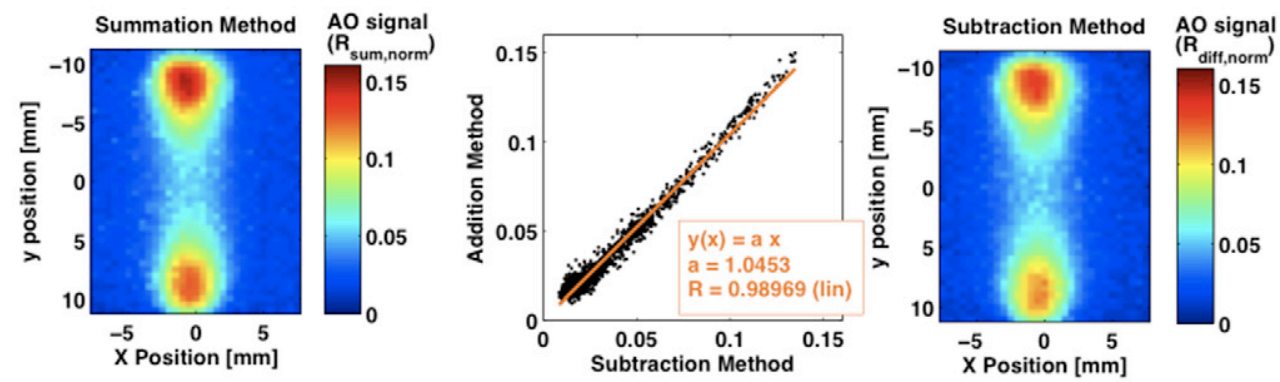

Figure 2. Estimated tagged fractions obtained with the summation method (left) and the subtraction method (right) compared against each other (center).
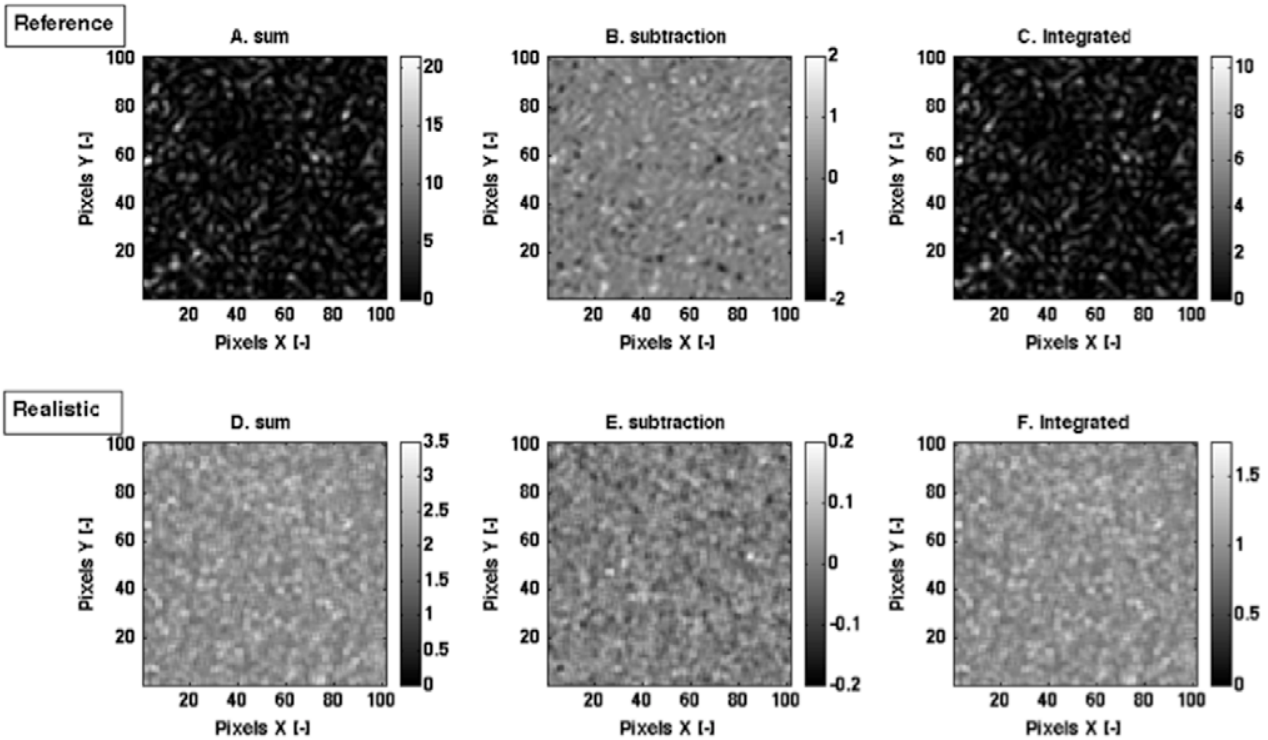

Figure 3. Two sets of cropped speckle patterns, the reference (top) and the realistic case (bottom), for the summated pattern (left), subtracted pattern (center) and integrated pattern that is used for the contrast method (right). Where the contrast values are near unity and 0.13 for the reference and realistic integrated speckle pattern, respectively.

\section{Results}

The maximum observed speckle contrast in this experiment was $c_{\max }=0.47$. A plot of the median of 6 measurements per position of $R_{\text {diff,Norm }}$ (right) and $R_{\text {sum,Norm }}$ (left) in figure 2 shows that the plots are nearly identical.

We plotted the pixel values against each other and found a near unity slope (figure 2 center plot).

To illustrate the speckle patterns obtained from the simulation, in figure 3 we show the reference and realistic case as described above of 3 (sum, subtraction and contrast) speckle patterns, the first speckle pattern is obtained by adding two instantaneous speckle patterns at two opposite ultrasound phases, the second by subtraction of these phases, and the third is obtained by integration of the speckle field over a complete US cycle. The data comes from the simulated speckle pattern for the ideal case that is used as reference, the second set comes 


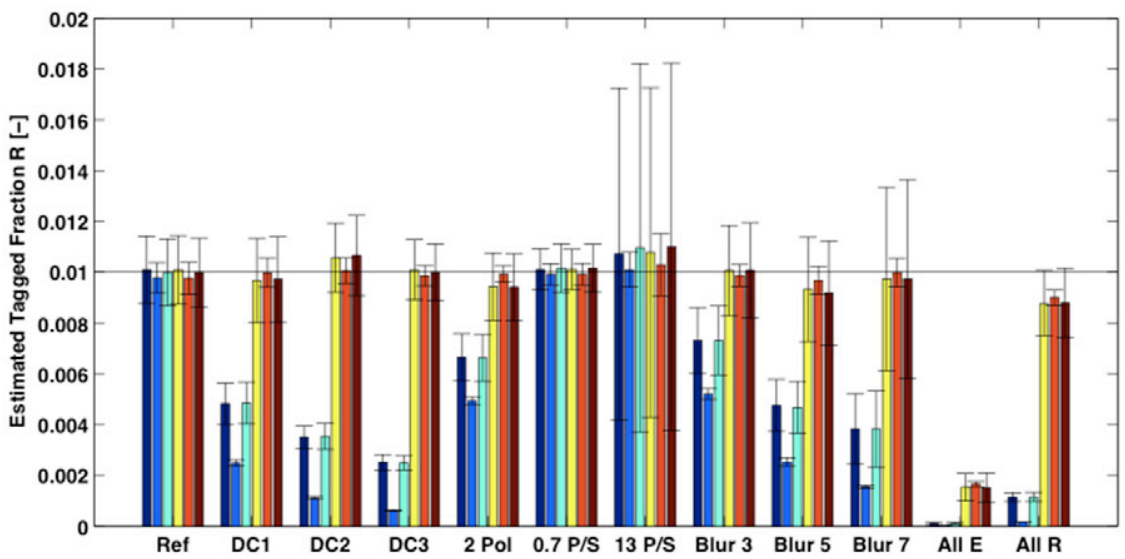

Figure 4. Estimated tagged fractions from simulated speckle patterns before and after correction, for the 3 methods for several contrast lowering mechanisms. Per mechanism 6 bars are given, from left to right: summation, subtraction, and speckle contrast method, followed by summation, subtraction, and speckle contrast method corrected with $c_{\max }$.

from the simulation for the more realistic case. Thus the speckle contrast of the integrated speckle pattern of the reference set is near unity and for the integrated realistic case $c=0.13$.

First of all we see in figure 4 that all of the values of the reference reproduce the set value of 0.01 for the tagged fraction. Figure 5 shows a speckle contrast of 1 for this reference. After correction the DC value has no influence on the estimated tagged fraction. The case of two polarizations is also predicting the correct value after correction. As shown in figure 5 the speckle contrast for this case is $1 / 2 \sqrt{ } 2$ as expected. The speckle contrast for the different speckle sizes is also unity because of the point sampling. The error bars in figure 4 are large in these cases because the amount of speckles on the camera decreases with the speckle size. The spatial averaging has a clear effect on the speckle contrast shown in figure 5 and causes a slight underestimation of the tagged fraction in figure 4. In the case of combined extreme distortions ('All E') the speckle contrast is very low and apparently the standard deviation of the contrast distorting function $\gamma$ in equation (17) is too large to successfully recover the tagged fraction after correction. But the more realistic combined case ('All R'), for which the distortions consist of addition of a DC value equal that of the average intensity and blurring by convolution of $3 \times 3$ pixels and two polarizations, only gives a small underestimation of the tagged fraction.

\section{Discussion and conclusion}

We derived and validated a correction method to compensate $\mathrm{AO}$ measurements for reduced speckle contrast induced by non-ideal properties of the set-up. This compensation makes measurements comparable between different setups. By recording speckle patterns at two opposite phases of the ultrasound we are able to extract an AO signal by subtraction or addition of the two speckle patterns, see equations (6) and (7), in which the maximum obtainable speckle contrast $c_{\max }$ is used to correct the measured result for speckle contrast reductions. The maximum obtainable contrast $c_{\max }$ for a given setup must be determined experimentally. This can be done by performing a measurement without applying ultrasound on a very stable phantom.

We have shown (see figure 2) that tagged fractions obtained with the summation method and subtraction method in an experiment are in agreement with each other after applying our correction method. The slope between these methods was found to be 1.04 in figure 2 and is 


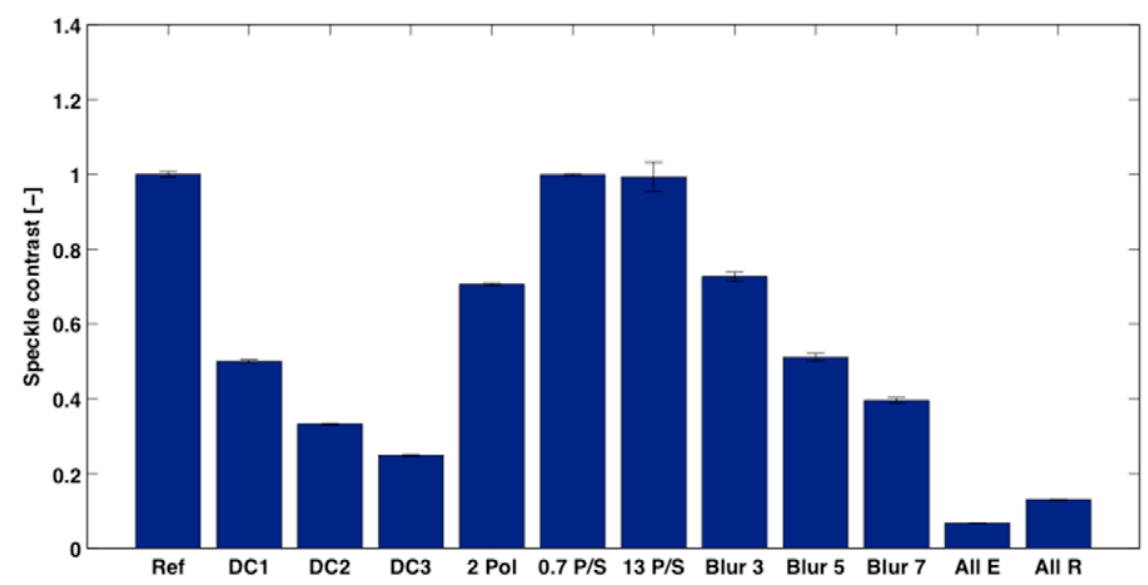

Figure 5. The speckle contrast values obtained from the simulations for the set of speckle contrast lowering mechanisms, working on the speckles without ultrasound modulation.

highly influenced by the accuracy of measurement of the maximum achievable speckle contrast estimation $c_{\max }$ that is used as normalization parameter.

The analytical expression of equation (17) shows that the reduction in speckle contrast after correction gives virtually the same value as predicted by the reference. This expression was tested with a set of simulations that show to which extent the type of speckle distortion and its magnitude influence the reliability of the correction. For a realistic case where the tagged fraction $R$ was set to 0.01 the correction has shown to work and the set value of the tagged fraction $R$ was restored with little deviation, see figure 4 . We expect that this correction method is valid for a wide range of tagged fraction values and that we are limited by noise at the lower end of the range. And at the higher end of the range we are limited by the distribution of phase modulation amplitudes over the aperture (Resink et al 2015).

Because the AO values of a measurement with low contrast speckle patterns can be corrected to the values that we expect for ideal high contrast speckle patterns, we can determine the fraction of tagged light $R$ in a speckle pattern. As a consequence we know the mean square of the phase modulation amplitude linking the measureable quantities to physical changes inside the medium (Resink et al 2015). This all makes our AO method better reproducible in case of different speckle contrast values between measurements and setups. In this way, quantifying AO signals becomes robust against influence DC offsets, finite pixels size differences, small camera readout noise and so on. When the influence is very large and a great degree of accuracy is required the standard deviation of the distortion pattern $\gamma$ must be determined by simulation of speckle fields with a guessed $\gamma$ such that it matches measured speckle statistics of static reference objects.

This work makes the AO part of our strategy of combining PA and AO more quantitative and shows that the two-pulse summation method is performing the same way as the speckle contrast method. The summation method and the subtraction method are compatible with pulsed lasers used for photoacoustic measurements. In figure 4 , the estimation of tagged fraction $R$ on simulated speckle patterns with the subtraction method shows less noise than the speckle contrast and summation method in figure 4 and may be the preferred strategy, although technically more challenging than the summation method since it requires that two speckle patterns of two laser pulses fired within the same US pulse are acquired separately in two separate camera frames. 


\section{Acknowledgment}

This work is supported by the Foundation for Fundamental Research on Matter (FOM), which is part of the Netherlands Organisation for Scientific Research (NWO), under grant 09NIG01.

\section{References}

Briers D, Duncan D D, Hirst E, Kirkpatrick S J, Larsson M, Steenbergen W, Stromberg T and Thompson O B 2013 Laser speckle contrast imaging: theoretical and practical limitations J. Biomed. Opt. 18066018

Daoudi K, Hussain A, Hondebrink E and Steenbergen W 2012 Correcting photoacoustic signals for fluence variations using acousto-optic modulation Opt. Express 20 14117-29

Hussain A, Daoudi K, Hondebrink E and Steenbergen W 2014 Mapping optical fluence variations in highly scattering media by measuring ultrasonically modulated backscattered light J. Biomed. Opt. 19066002

Leveque S, Boccara A C, Lebec M and Saint-Jalmes H 1999 Ultrasonic tagging of photon paths in scattering media: parallel speckle modulation processing Opt. Lett. 24 181-3

Li J, Ku G and Wang L H V 2002 Ultrasound-modulated optical tomography of biological tissue by use of contrast of laser speckles Appl. Opt. 41 6030-5

Ramirez-San-Juan J C, Mendez-Aguilar E, Salazar-Hermenegildo N, Fuentes-Garcia A, Ramos-Garcia R and Choi B 2013 Effects of speckle/pixel size ratio on temporal and spatial speckle-contrast analysis of dynamic scattering systems: implications for measurements of bloodflow dynamics Biomed. Opt. Express 4 1883-9

Resink S G, Boccara A C and Steenbergen W 2012 State-of-the art of acousto-optic sensing and imaging of turbid media J. Biomed. Opt. 17040901

Resink S G, Hondebrink E and Steenbergen W 2014a Solving the speckle decorrelation challenge in acousto-optic sensing using tandem nanosecond pulses within the ultrasound period Opt. Lett. 39 6486-9

Resink S G, Hondebrink E and Steenbergen W 2014b Towards acousto-optic tissue imaging with nanosecond laser pulses Opt. Express 22 3564-71

Resink S G and Steenbergen W 2015 Tandem-pulsed acousto-optics: an analytical framework of modulated high-contrast speckle patterns Phys. Med. Biol. 604371

Thompson O, Andrews M and Hirst E 2011 Correction for spatial averaging in laser speckle contrast analysis Biomed. Opt. Express 2 1021-9

Zemp R, Sakadzic S and Wang L V 2006 Stochastic explanation of speckle contrast detection in ultrasound-modulated optical tomography Phys. Rev. E 73061920 